\title{
Changes in muscle activity of the abdominal muscles according to exercise method and speed during dead bug exercise
}

\author{
Byeong-Gwon Yun ${ }^{a}$, Seung-Joo Lee ${ }^{b}$, Hyun-Jeong So ${ }^{b}$, Won-Seob Shin ${ }^{b, c}$ \\ ${ }^{a}$ Department of Physical Therapy, VHS Medical Center, Seoul, Republic of Korea \\ ${ }^{b}$ Department of Physical Therapy, Graduate School of Health and Medicine, Daejeon University, Daejeon, Republic of Korea \\ 'Department of Physical Therapy, College of Health and Medical Science, Daejeon University, Daejeon, Republic of Korea
}

\begin{abstract}
Objective: There are many types of exercises with upper and lower-limb action for activation of abdominal muscles for trunk stabilization. A comparison of the different exercise methods been very useful to enhance the result from the exercise for treatment. The purpose of this study was to investigate through surface electromyography (EMG) the changes in abdominal muscle activity during the performance of three different dead-bug exercise methods performed at three different speeds.

Design: Cross-sectional study.

Methods: The subjects were 30 healthy adults (13 males and 17 females). We instructed the subjects to perform three different dead-bug exercises. We also applied three different speeds to the dead-bug exercises; $60 \mathrm{bpm}, 90 \mathrm{bpm}$, and $120 \mathrm{bpm}$ with use of a metronome. The assessment of EMG was percentage of maximal voluntary isometric contraction on the rectus abdominis (RA), external oblique (EO), and internal oblique (IO).

Results: EMG activation of the RA, EO and IO muscles was significantly greater at the higher speed $(p<0.05)$. There was a significant increase in abdominal muscle activity during the dead-bug exercise performed with both the upper and lower extremities compared to that with only the upper extremity or the lower extremities $(p<0.05)$.

Conclusions: These findings demonstrate that the performance of the dead-bug exercise with both the upper and lower extremities combined at a high speed is more effective compared to other exercises. Therefore, it is suggested that more favourable and effective outcomes may occur when the type and speed of the exercise is chosen appropriately.
\end{abstract}

Key Words: Electromyography, Exercise movement techniques, Exercise therapies

\section{Introduction}

Most exercise programs for back pain management highlights mostly improving trunk muscle strength, endurance, and functional capacity, however, recent studies have emphasized improving motor control of the trunk muscles $[1,2]$. Motor control of the trunk muscles includes orderly mobilization of the trunk muscles and activation of the core muscles involved in providing stability, but motor control imbalances may lead to spinal instability, which is the most im- portant cause of low back pain [3]. Intervention to enhance trunk stability includes mostly strengthening the abdominal and erector spinae muscles. Strengthening the trunk muscles of the spine provides dynamic stability of the spine segments, which helps to reduce spinal dysfunction [4].

Trunk stabilization exercises to strengthen the trunk muscle includes performing curl-ups in a supine position [5], quadruped exercise where the arms and legs are lifted while assuming a quadruped position [6], and the dead-bug exercise where the arms and legs are alternately crossed while

Received: 27, February, 2017 Revised: 13 March, 2017 Accepted: 13 March, 2017

Corresponding author: Won-Seob Shin

Department of Physical Therapy, College of Health and Medical Science, Daejeon University, 62 Daehak-ro, Dong-gu, Daejeon 34520, Republic of Korea Tel: 82-42-280-2290 Fax: 82-42-280-2295 E-mail: shinws@dju.kr

(c) This is an Open-Access article distributed under the terms of the Creative Commons Attribution Non-Commercial License (http://creativecommons.org/licens es/by-nc/4.0) which permits unrestricted non-commercial use, distribution, and reproduction in any medium, provided the original work is properly cited.

Copyright @ 2017 Korean Academy of Physical Therapy Rehabilitation Science 
in a supine position [6]. These exercise methods enhances the abdominal muscles which are important for providing trunk stabilization during trunk and upper extremity movements, and are effective in promoting overall function $[5,6]$. Of these exercises, the dead-bug exercise is an effective exercise for promoting stabilization of the trunk and pelvis and is performed by alternately moving the arms and legs while maintaining the abdominal draw-in maneuver, which activates the rectus abdominis and oblique muscles.

An individualized exercise program based on the individual's condition and capabilities is necessary when trying to achieve an exercise goal by having an appropriate combination of exercise intensity and frequency levels [7]. Exercise intensity is established according to the resistance or weight applied in a single perform and changes in frequency, momentum, resting periods, repetition rates can be made as a way to adjust the exercise intensity [8].

Souza et al. [6] reported that during the performance of the dead-bug exercise, resistance is applied through the weight of the arms and legs, and by conducting a study on investigating the effects of increasing the exercise load on abdominal muscle activity, it had been confirmed that activity was increased with gradual increases in resistance [6]. As a method of adjusting the exercise intensity, various postures and exercise methods are being used in studies increase the effectiveness of the dead-bug exercise that may lead to changes in trunk stability [9].

Therefore, the purpose of this study was to compare various methods that can be used to increase the effectiveness of the general dead-bug exercise on trunk stability. This study also attempted to investigate the difference in the effects of performing different intensities of the dead-bug exercise through the incorporation of upper and lower extremity movements as well as exercising at various speeds on abdominal muscle activity.

\section{Methods}

\section{Subjects}

Thirty healthy college students (13 males, 17 females) from Daejeon University were recruited for the study. Those who have had an orthopedic or neurological problem within the last six months and those who had experienced pain with exercises were excluded from the study. All subjects provided an informed consent and volunteered for the study after being presented with the explanation of the study purpose and methods.

\section{Procedure}

\section{Dead-bug exercise}

The dead-bug exercise was performed with the subjects in supine position with the spine maintained in neutral position [6]. Prior to performing the exercise, subjects were asked to slowly pull the abdomen toward the spine as a training method to perform the abdominal drawing-in maneuver [10]. This is reported to be an effective exercise method in providing spinal stabilization [11]. A biofeedback device (Stabilizer; Chattanooga Group Inc., Hixson, TN, USA) with a pressure of 40 to $70 \mathrm{mmHg}$ was maintained at the level below the 5 th lumbar vertebrae, which was used to confirm and maintain trunk stabilization during the abdominal drawing-in maneuver $[12,13]$.

\section{Changes in exercise methods}

The dead-bug exercise was divided into three different exercise methods. The starting position for the first deadbug exercise method included only the upper extremities with the shoulder flexed to 90 degrees and with the knees straight up while lying in supine. The starting position for the second dead-bug exercise method included only the lower extremities with the upper extremities laid onto the ground and with the hip joint and knee joints in 90 degrees flexion. The starting position for the third dead-bug exercise method included movement of both the upper and lower extremities with the shoulders, hip joint and knees flexed to 90 degrees (Figure 1).

\section{Changes in exercise speed}

The dead-bug exercises were divided into three different speeds. Exercise speeds were guided with use of a metronome. For each type of dead-bug exercise, the upper and lower extremity movements were performed at speeds of 60 bpm, $90 \mathrm{bpm}$, and $120 \mathrm{bpm}$. Guidance was provided by an evaluator to ensure that each speed was accurately maintained, and if the subjects were unable to continuously perform the exercises, a rest period was provided prior to the resumption of the exercise.

\section{Outcome measures}

Surface electromyography (EMG) was used to investigate the muscle activity of the rectus abdominis, external oblique, and internal oblique muscles. A four-channel portable EMG system (LXM3204; Laxtha Inc., Daejeon, Korea) was used to collect data of the muscle activities of the right 

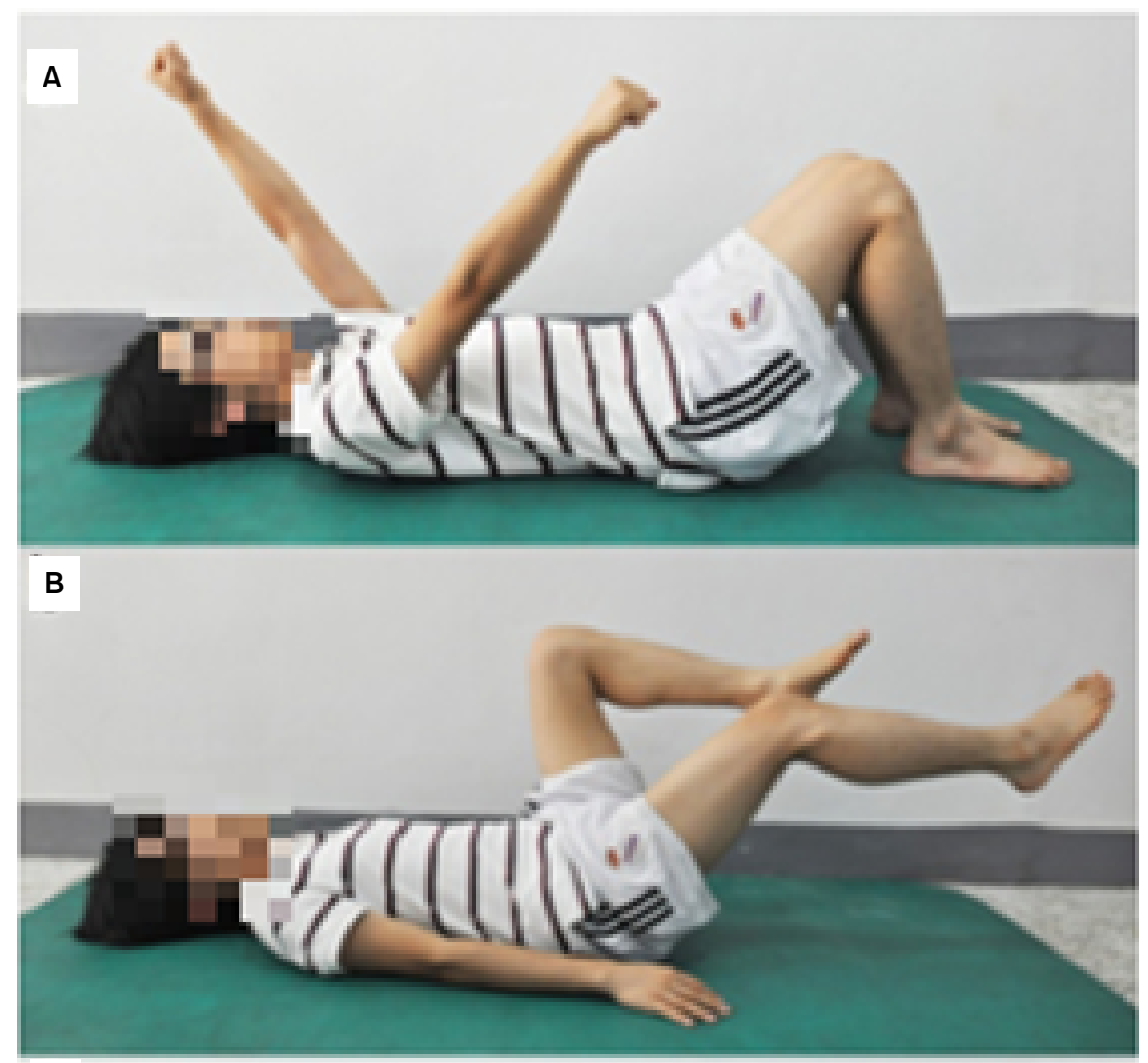

C

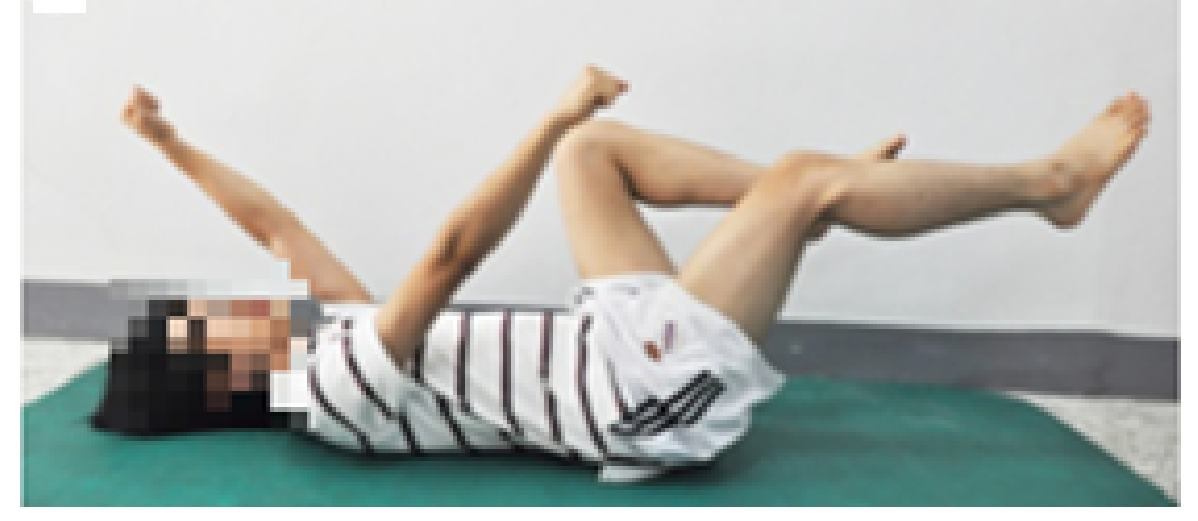

Figure 1. (A) Upper extremity dead-bug exercise. (B) Lower extremity dead-bug exercise. (C) Upper and lower extremity dead- bug exercise. rectus abdominis, external abdominal oblique, and internal abdominal oblique muscles.

After marking the electrode sites for each muscle, each site was rubbed 3 to 5 times with sandpaper in order to minimize resistance by removing the horny layer of the skin. The skin was sterilized with rubbing alcohol afterwards. For the rectus abdominis muscle, electrodes were attached $2 \mathrm{~cm}$ laterally and distally from the umbilicus. For the external abdominal oblique muscle, the electrodes were attached between the end of the rib and the anterior superior iliac spine (ASIS) [14]. For the internal abdominal oblique muscle, electrodes were attached parallel to and approximately $2 \mathrm{~cm}$ downwards from the ASIS. The ground electrode was attached onto the right ASIS. A sampling rate of 1,024 Hz was used and the collected data was processed using the TeleScan 3.01 program (Laxtha Inc.). The maximal voluntary isometric contraction (MVIC) was measured to standardize the action potential of each muscle. To encourage maximal activation of the rectus abdominis, subjects were instructed to perform trunk flexion from the supine position, bend and rotate the trunk to the opposite side for the external oblique, and lateral trunk rotation for the internal oblique 
muscle [15]. The evaluator provided resistance toward the opposite direction of each muscle position to the induce maximal isometric contraction of each muscle [16]. The MVIC was assessed 3 times for 5 seconds, with a 2-minute rest period provided between each MVIC assessment session [17]. With the exclusion of the first and fifth second, the mean values from the middle 3 seconds were calculated. After signals had undergone full wave rectification, it was processed into the root mean square.

After performing the abdominal drawing-in maneuver for the dead-bug exercise, once the patient was at a state of being able to maintain sufficient pressure they had included the arm movements. Three different types of arm movements were performed by the patient in random order in addition to three different speeds for each type of exercise used in random order as well. All exercises were performed for $10 \mathrm{sec}-$ onds, however, excluding the first and last three seconds, only values from the middle four seconds were used. In addi-

Table 1. General characteristics of subjects $(\mathrm{N}=30)$

\begin{tabular}{lccc}
\hline Characteristic & Male $(\mathrm{n}=13)$ & Female $(\mathrm{n}=17)$ & Total \\
\hline Age $(\mathrm{yr})$ & $23.54(1.33)$ & $22.12(5.43)$ & $22.73(1.33)$ \\
Heigth $(\mathrm{cm})$ & $173.60(7.25)$ & $160.85(38.16)$ & $166.37(8.60)$ \\
Weigth $(\mathrm{kg})$ & $70.46(10.01)$ & $51.85(13.56)$ & $59.92(12.23)$ \\
\hline
\end{tabular}

Values are presented as mean (SD). tion, subjects performed the exercises three times according to each speed type, and mean values were obtained. To avoid fatigue, a 2-minute rest period was provided between each exercise [17].

\section{Data and statistical analysis}

The results of this study were analyzed with the IBM SPSS Statistics ver. 20.0 (IBM Co., Armonk, NY, USA). Normal distribution was confirmed with the KolmogorvSmirnov test. The two-way repeated measures ANOVA was used to analyze the EMG results from the abdominal muscles during the performance of three different exercises with three various speeds. In addition, the Bonferroni was used for post-hoc analysis. The significance level was set at $\alpha=$ 0.05 .

\section{Results}

\section{General characteristics of subjects}

The general characteristics of subjects are presented in Table 1 . There were 30 subjects ( 13 males, 17 females) with an average height of $166.37 \mathrm{~cm}$ and an average weight of $59.92 \mathrm{~kg}$ (Table 1).

Table 2. Comparison of the three group in pre-post muscle activity

\begin{tabular}{|c|c|c|c|c|}
\hline Variable & $60 \mathrm{bpm}$ & 90 bpm & $120 \mathrm{bpm}$ & $\mathrm{F}$ \\
\hline \multicolumn{5}{|c|}{ RA (\%MVIC) } \\
\hline UE & $8.88(7.17)$ & $10.15(11.26)$ & $11.03(11.02)$ & 3.010 \\
\hline LE & $28.79(15.66)^{\mathrm{a}}$ & $31.88(16.59)^{\mathrm{a}, \mathrm{b}}$ & $37.06(19.65)^{\mathrm{a}, \mathrm{b}, \mathrm{c}}$ & $20.893^{*}$ \\
\hline U/LE & $31.52(14.91)^{\mathrm{a}}$ & $34.45(16.05)^{\mathrm{a}, \mathrm{b}}$ & $37.52(17.57)^{\mathrm{a}, \mathrm{b}, \mathrm{c}}$ & $7.989^{*}$ \\
\hline $\mathrm{F}$ & $29.262^{*}$ & $28.842^{*}$ & $29.299^{*}$ & \\
\hline \multicolumn{5}{|c|}{ EO (\%MVIC) } \\
\hline UE & $11.85(6.93)$ & $13.71(10.06)$ & $14.44(7.27)^{\mathrm{b}}$ & $17.064^{*}$ \\
\hline LE & $35.75(16.60)^{\mathrm{a}}$ & $39.53(17.83)^{\mathrm{a}, \mathrm{b}}$ & $44.33(18.83)^{\mathrm{a}, \mathrm{b}, \mathrm{c}}$ & $37.178^{*}$ \\
\hline $\mathrm{U} / \mathrm{LE}$ & $35.81(15.44)^{\mathrm{a}}$ & $40.70(18.95)^{\mathrm{a}, \mathrm{b}}$ & $48.11(21.40)^{\mathrm{a}, \mathrm{b}, \mathrm{c}}$ & $29.192^{*}$ \\
\hline $\mathrm{F}$ & $66.235^{*}$ & $83.223^{*}$ & $95.840^{*}$ & \\
\hline \multicolumn{5}{|c|}{ IO (\%MVIC) } \\
\hline UE & $11.69(9.55)$ & $15.26(13.18)^{\mathrm{b}}$ & $16.51(13.19)^{\mathrm{b}}$ & $7.349^{*}$ \\
\hline LE & $33.94(14.09)^{\mathrm{a}}$ & $40.07(16.30)^{\mathrm{a}, \mathrm{b}}$ & $47.62(17.38)^{\mathrm{a}, \mathrm{b}, \mathrm{c}}$ & $64.039^{*}$ \\
\hline $\mathrm{U} / \mathrm{LE}$ & $36.61(14.56)^{\mathrm{a}}$ & $42.07(16.58)^{\mathrm{a}, \mathrm{b}}$ & $50.03(18.09)^{\mathrm{a}, \mathrm{b}, \mathrm{c}}$ & $24.357^{*}$ \\
\hline $\mathrm{F}$ & $23.910^{*}$ & $15.839^{*}$ & $24.117^{*}$ & \\
\hline
\end{tabular}

Values are presented as mean (SD).

RA: rectus abdominis, UE: upper extremity, LE: lower extremity, EO: external abdominal oblique, IO: internal abdominal oblique.

${ }^{\mathrm{a}}$ Significant differences compared to upper extremity, ${ }^{\mathrm{b}}$ Significant difference compared to $60 \mathrm{bpm}$, ${ }^{\mathrm{c}}$ Significant difference between 90 bpm and 120 bpm.

${ }^{*} p<0.05$. 


\section{A comparison of abdominal muscle activity according to various exercise methods and speed}

There was a statistically significant difference in abdominal muscle activity according to the dead-bug exercise with extremities Post-hoc analysis showed that there was a significant increase in abdominal muscle activity with the lower extremity and the upper and lower extremity dead-bug exercise group compared to the upper extremity dead-bug exercise group $(p<0.05)$, but there was no significant difference between the lower extremity with the upper and lower extremity dead-bug exercise group.

There was a significant difference in abdominal muscle activity between the lower extremity and the upper and lower extremity dead-bug exercise performed at $60 \mathrm{bpm}, 90$ bpm, and $120 \mathrm{bpm}$. In addition, post-hoc analysis showed that there was a significant increase in abdominal muscle activity with exercise performance at a high speed compared to a low speed $(p<0.05)$.

However, there was no significant difference in rectus abdominis muscle activity according to difference speeds in the lower extremity dead-bug exercise group. For the external abdominal oblique muscle, significant differences were observed only at $60 \mathrm{bpm}$ and $120 \mathrm{bpm}$. Although there was a significant difference in internal abdominal oblique muscle activity with the dead-bug exercise performed at 90 bpm and $120 \mathrm{bpm}$ compared to $60 \mathrm{bpm}(p<0.05)$, there was no significant difference between the exercises performed at $90 \mathrm{bpm}$ and $120 \mathrm{bpm}$ (Table 2).

\section{Discussion}

The purpose of this study was to investigate the effects of performing the dead-bug exercises using three different methods and speeds on abdominal muscle activity. As a method used in the clinic to enhance trunk stability, the biofeedback is used to confirm whether the abdominal draw-in maneuver is being performed appropriately. From this study, when comparing the three different dead-bug exercise methods, it had been found that the abdominal muscle activity was the highest with the upper and lower extremity deadbug exercise. Souza et al. [6] observed a significant increase in rectus abdominis and oblique muscle activity when subjects performed the dead-bug exercise with the additional weight of the upper and lower extremities

Increases in muscle activity with increase weight loads occur due to the stimulation of the supplementary motor area of the cerebral cortex [18]. Therefore, it is considered that increases in abdominal muscle activity se occur especially with lower extremity movements since the weight of the lower extremities are generally greater than the upper extremities. McGill and Karpowicz [9] compared the abdominal muscle activity according to the different types of Bird-dog exercise performed in a quadruped position involved with the lifting of the opposite upper and lower extremities. Although increases in rectus abdominis, oblique muscle activities had been observed during the performance of the bird-dog exercise with the upper extremities, lower extremities, or combined with both upper and lower extremities, there was no significant difference in abdominis muscle activity according to each specific type of exercise. Therefore, further pre and post studies are needed to compare the multifidus muscle activity according to different bird-dog exercise methods.

This study showed that when performing the dead-bug exercises with three different speeds, there was increased abdominal muscle activity with the upper and lower extremity dead-bug exercise performed at high speed. Hodges and Richardson [19] stated that with faster the upper extremity movements, there was a more significantly increased trunk and deltoid muscle activity while in contrast, the slower the upper extremity movements, the lower the muscle activity of the trunk and deltoid muscle [19]. McGill and Karpowicz [9] compared the abdominal muscle activity while performing a slow dead-bug exercise versus a fast, plyometric-type exercise. While the MVIC values of the abdominal, external oblique and internal oblique were $6 \%$, $8 \%$, and $5 \%$ during the slow dead-bug exercise, the MVIC values were $53 \%, 26 \%$, and $42 \%$ during the high-speed plyometric method of exercise and showed a significant increase in abdominal muscle activity [9].

It is considered that the reason for the increased abdominal muscle activity is that with higher upper extremity movement speeds, the abdominal muscle response is increased in order to maintain trunk stability.

A limitation of this study was that although a metronome was used with use of the EMG, it was difficult to identically perform the dead-bug exercise movements along with the metronome. Therefore, further studies should include performing a motion analysis in order to accurately measure the movements.

Through this study, it has been confirmed that the muscle activity of the abdominal muscles were enhanced by performing the dead-bug exercise for trunk stability at a higher 
speed. Through these results, it is suggested that the dead-bug exercises can be used to increase abdominal muscle activity to promote trunk stability by using different exercise methods and various speeds.

\section{Conflict of Interest}

The authors declared no potential conflicts of interest with respect to the authorship and/or publication of this article.

\section{References}

1. Luoto S, Aalto H, Taimela S, Hurri H, Pyykkö I, Alaranta H. One-footed and externally disturbed two-footed postural control in patients with chronic low back pain and healthy control subjects. A controlled study with follow-up. Spine (Phila Pa 1976) 1998;23:2081-9; discussion 2089-90.

2. Marshall P, Murphy B. The validity and reliability of surface EMG to assess the neuromuscular response of the abdominal muscles to rapid limb movement. J Electromyogr Kinesiol 2003; 13:477-89.

3. Comerford MJ, Mottram SL. Movement and stability dysfunction: contemporary developments. Man Ther 2001;6:15-26.

4. Panjabi MM. Clinical spinal instability and low back pain. J Electromyogr Kinesiol 2003;13:371-9.

5. Liebenson C. Spinal stabilization training: the transverse abdominus. J Bodyw Mov Ther 1998;2:218-23.

6. Souza GM, Baker LL, Powers CM. Electromyographic activity of selected trunk muscles during dynamic spine stabilization exercises. Arch Phys Med Rehabil 2001;82:1551-7.

7. Kraemer WJ, Ratamess NA. Fundamentals of resistance training: progression and exercise prescription. Med Sci Sports Exerc 2004;36:674-88.

8. Kraemer WJ, Ratamess N, Fry AC, Triplett-McBride T, Koziris LP, Bauer JA, et al. Influence of resistance training volume and periodization on physiological and performance adaptations in collegiate women tennis players. Am J Sports Med 2000;28:626-33.

9. McGill SM, Karpowicz A. Exercises for spine stabilization: motion/motor patterns, stability progressions, and clinical technique. Arch Phys Med Rehabil 2009;90:118-26.

10. Vasseljen O, Fladmark AM. Abdominal muscle contraction thickness and function after specific and general exercises: a randomized controlled trial in chronic low back pain patients. Man Ther 2010;15:482-9.

11. Goldby LJ, Moore AP, Doust J, Trew ME. A randomized controlled trial investigating the efficiency of musculoskeletal physiotherapy on chronic low back disorder. Spine (Phila Pa 1976) 2006;31:1083-93.

12. Choi HS, Shim YJ, Shin WS. Comparison on postural control between abdominal draw-in maneuver and abdominal expansion maneuver in persons with stroke. Phys Ther Rehabil Sci 2016;5:113-9.

13. Ko YJ, Ha HG, Jeong J, Lee WH. Variations in lateral abdominal muscle thickness during abdominal drawing-in maneuver in three positions in a young healthy population. Phys Ther Rehabil Sci 2014;3:101-6.

14. Ng JK, Kippers V, Richardson CA. Muscle fibre orientation of abdominal muscles and suggested surface EMG electrode positions. Electromyogr Clin Neurophysiol 1998;38:51-8.

15. Lee J, Jeong K, Lee H, Shin J, Choi J, Kang SB, et al. Comparison of three different surface plank exercises on core muscle activity. Phys Ther Rehabil Sci 2016;5:29-33.

16. Sapsford RR, Hodges PW, Richardson CA, Cooper DH, Markwell SJ, Jull GA. Co-activation of the abdominal and pelvic floor muscles during voluntary exercises. Neurourol Urodyn 2001;20:31-42.

17. Ng JK, Richardson CA, Parnianpour M, Kippers V. EMG activity of trunk muscles and torque output during isometric axial rotation exertion: a comparison between back pain patients and matched controls. J Orthop Res 2002;20:112-21.

18. Oda S, Shibata M, Moritani T. Force-dependent changes in movement-related cortical potentials. J Electromyogr Kinesiol 1996;6:247-52.

19. Hodges PW, Richardson CA. Altered trunk muscle recruitment in people with low back pain with upper limb movement at different speeds. Arch Phys Med Rehabil 1999;80:1005-12. 